\title{
CORRIGENDUM
}

\author{
A. Hua-Van · J. A. Pamphile $\cdot$ T. Langin
}

M.-J. Daboussi

\section{Transposition of autonomous and engineered impala transposons in Fusarium oxysporum and a related species}

Published online: 15 December 2000

(C) Springer-Verlag 2001

\section{Mol Gen Genet (2001)}

Due to a technical error part C of Fig. 4 was omitted. The whole figure is printed below.

The online version of the original article can be found at http:// dx.doi.org/10.1007/s004380000395

A. Hua-Van $(\bowtie)^{1} \cdot$ J. A. Pamphile ${ }^{2} \cdot$ T. Langin ${ }^{3} \cdot$ M.-J. Daboussi Institut de Génétique et Microbiologie,

Université Paris-Sud, F-91405 Orsay Cedex, France

E-mail: daboussi@igmors.u-psud.fr

Tel.: + 33-1-69156213

Fax: + 33-1-69157006

Present addresses:

${ }^{1}$ Department of Biological Sciences,

University of Notre Dame, Notre Dame, IN 46556, USA

${ }^{2}$ Universidade Estadual de Maringá, Departamento de Biologica Celular e Genetica, Maringá, PR, Brazil

${ }^{3}$ Institut de Biotechnologie des Plantes, Université Paris-Sud, 91405 Orsay Cedex, France

$\begin{array}{llr}\text { wt } & \text { CCTATCCCAT } & \text { ACTCT } \\ \text { niaD160 } & \text { CCTATCCCATAcagt. .imp160. actgTACTCT }\end{array}$

A Fusarium oxysporum

Fo5nia13 + imp160

$\mathrm{n}=5$
$\mathrm{n}=1$
$\mathrm{n}=1$

B Fusarium moniliforme

\begin{tabular}{|c|c|}
\hline \\
\hline \\
\hline \multicolumn{2}{|r|}{ 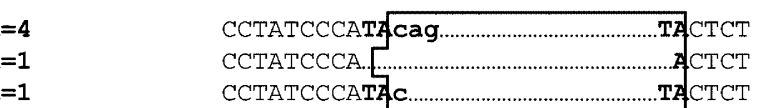 } \\
\hline \multicolumn{2}{|c|}{$\mathrm{C}_{\text {Reinsertion sites }}$} \\
\hline & TGTCCATCTACag. . imp. . ctgTATACG \\
\hline & GTTGATAGTACag. . imp. . ctgTATTCTC \\
\hline
\end{tabular}

Fig. 4A-C Structure of impala excision sites in $F$. oxysporum revertants $(\mathbf{A})$ and in $F$. moniliforme revertants $(\mathbf{B})$. Sequences of the wild-type gene (wt) and the original insertion mutant (niaD160) are given at the top. $\mathrm{n}=$ number of revertants with the indicated sequence. Bold capitals: duplicated target site TA. Bold lower cases: impala ends. Nucleotide insertion, relative to the wild type, are framed. C. Sequences of two reinsertion sites. impala sequences are in italics and the duplicated target sites are in bold 